\title{
Audit of catheter urine culture requests
}

\author{
N Manek, E Napier Rees
}

\begin{abstract}
An audit to assess the appropriateness of catheter urine culture requests was carried out for a period of one month. The requests were followed up by members of the Infection Control Team at ward level. The laboratory report had no impact on the removal of the catheter in asymptomatic or symptomatic patients, although the reports did aid antibiotic prescribing in symptomatic patients.
\end{abstract}

Indwelling bladder catheters are the major source of hospital acquired infections. ${ }^{1}$ The initial infection is generally due to Staphylococcus epidermis, Streptococcus faecalis, Escherichia Coli or Proteus spp, ${ }^{2}$ although the longer the catheter remains indwelling, the greater the variety of organisms that will appear in the urine. Bacteriological monitoring of catheter urine is frequently requested in many patients, even though most serious complications are associated with a symptomatic patient. The laboratory processing of catheter urines is time consuming, laborious, and costly. A well defined policy for requesting catheter urine culture would aid the clinician, the laboratory, and avoid wasting resources.

\section{Methods and results}

To define guidelines, catheter urine culture requests from inpatients were followed up at ward level for a period of one month. The requests were placed into two categories: category 1 , requests from symptomatic patients with relevant clinical details, such as fever or septicaemia; and category 2, requests from asymptomatic patients with irrelevant clinical details or labelled as routine, such as myocardial infarcation, stroke.

A total of 146 requests were received from inpatients. The urine samples were cultured using the strip test method. ${ }^{3}$ Traditionally, significant bactiuria defined as more than $10^{5}$ colony forming units of bacteria/ml of urine differentiates between urinary tract infection and extraneous contamination. ${ }^{4}$ In this audit and other catheter studies ${ }^{5}$ the same numerical criterion has been used to provide a valid

Department, St George's Hospital, Stafiord

N Manek

E Napier Rees

Correspondence to:

Dr Manek, Department of

Clinical Microbiology,

Queen Elizabeth Hospital,

Queen Elizabeth Hospital,

Bdgbasto

Accepted for publication

4 July 1991

\begin{tabular}{|c|c|c|}
\hline & Category 1 & Category 2 \\
\hline Culture & Symptomatic patients & Asymptomatic patients \\
\hline $\begin{array}{l}\text { Pure } \\
\text { Mixed } \\
\text { No growth } \\
\text { Total }\end{array}$ & $\begin{array}{l}29 \\
11 \\
35 \\
75\end{array}$ & $\begin{array}{l}19 \\
16 \\
36 \\
71\end{array}$ \\
\hline
\end{tabular}

comparison between two groups of patients, even though infection with lower bacterial counts may occur in some catheterised patients. All requests were followed up on day 1 and day 3 by the infection control nurse to assess catheter management in terms of removal of catheter and start of antibiotic treatment (the laboratory report was issued on day 1).

Culture results from symptomatic and asymptomatic patients are shown in the table.

Nine symptomatic patients were excluded from the study as complete follow up was not possible. Follow up of the remaining patients, however, confirmed that in $38 \%$ the catheter had been removed before day 1 - that is, even before the laboratory report had reached the clinician. By day 3, $57 \%$ of catheters had been removed. It was impossible to remove the catheter in the remaining $43 \%$ of patients for many reasons. The decision to remove the catheter was not related to concern about a urinary tract infection, but was based on the medical condition of the patient. Antibiotic treatment was started in only $20 \%$ of symptomatic patients and the choice of antibiotic was initially based on a "blind guess" basis.

\section{Discussion}

This small study confirms our initial impressions that the culture of catheter urines is of value in symptomatic patients in whom antibiotic treatment may be considered. In asymptomatic catheterised patients the laboratory report had no impact on catheter removal and was of little value in catheter management. We would, therefore, suggest that a policy for requesting catheter urine cultures should be agreed with the clinicians. Such a policy should indicate that catheter urine specimens may be submitted to the laboratory only when there is a clinical indication for treatment, such as fever or septicaemia, and that this indication should be clearly marked on the request form.

We are grateful for the assistance of Mrs Barnett and Mrs Bennett, Microbiology Laboratory, Stafford.

1 Meers PD, Ayliffe GAJ, Emmerson AM, et al. National survey of infection in hospitals 1980. Part 2: Urinary tract infection. J Hosp Infect 1981(Suppl);2:23-8.

2 Bultitude MJ, Eykyn $S$. The relationship between the urethral flora and urinary infection in the catheterized urethral flora and urinary infection

3 Leigh DA, Williams JD. Methods for the detection of significant bacteriuria in large groups of patients. $J$ Clin Pathol 1964;17:498-503.

4 Kass EH. Asymptomatic infections of the urinary tract. Trans Assoc Am Phys 1956;69:56-64.

5 Stickler DJ. The role of antiseptics in the management of patients undergoing short-term indwelling bladder catheterization. J Hosp Infect 1990;16:89-108. 